\title{
Polytype distributions in low-defect zeolite beta crystals synthesized without an organic structure directing agent
}

Yukichi Sasaki,${ }^{* a}$ Yoko Yoshida, ${ }^{b}$ Craig A. J. Fisher, ${ }^{a}$ Takuji Ikeda,${ }^{c}$ Keiji Itabashi ${ }^{d, e}$ and Tatsuya Okubo $^{d}$

${ }^{a}$ Nanostructures Research Laboratory, Japan Fine Ceramics Center, 2-4-1 Mutsuno, Atsuta-ku, Nagoya, 456-8587, Japan.

${ }^{b}$ Aichi Center for Industry and Science Technology, 1267-1 Akiai, Yakusa-cho, Toyota, 4700356, Japan.

${ }^{c}$ Research Center for Compact Chemical Systems, National Institute of Advanced Industrial Science and Technology, 4-2-1 Miyagino-ku, Sendai, 983-8551, Japan.

${ }^{d}$ Department of Chemical System Engineering, The University of Tokyo, 7-3-1 Hongo, Bunkyoku, Tokyo 113-8656, Japan.

${ }^{e}$ Unizeo Co., Ltd., 7-3-1 Hongo, Bunkyo-ku, Tokyo 113-0033, Japan.

\section{AUTHOR INFORMATION}

\section{Corresponding Author}

Yukichi Sasaki, Fax: +81 52871 3599; Tel: +81 52871 3500; E-mail: sasaki@jfcc.or.jp 
ABSTRACT. Design of efficient and environmentally benign zeolitic materials at the nanoscale is becoming increasingly important for a range of industrial applications. Here we report a high resolution transmission electron microscopy investigation of the atomic level structure of zeolite beta synthesized without an organic structure-directing agent (OSDA). Direct visualization of zeolite beta crystals confirmed them to be essentially defect free, with domain boundaries the only disruption to the pore channel network. Direct visualization enabled the distribution of BEA and BEB polytype subdomains to be quantified, and the overall abundance ratio was calculated to be $\mathrm{BEA}: \mathrm{BEB}=9: 11$. By assuming this ratio corresponds to the probability of forming a new BEA or BEB layer on a (001) surface at the tip of a crystal, the experimentally observed polytype distributions can be described by a simple probability function, suggesting that epitaxial crystal growth occurs stochastically in a state close to chemical equilibrium.

KEYWORDS zeolite beta, OSDA-free, transmission electron microscopy, polytype

\section{Introduction}

Micron- and nanometer-sized zeolite crystallites are used in many catalytic and adsorption applications, and the efficient design of such materials and optimization of their synthesis parameters require an atomic level understanding of their crystal structures, growth mechanisms, and properties. ${ }^{1}$ In addition to the development of more powerful analytical and synthesis techniques, ${ }^{1,2}$ theoretical modeling is playing an increasingly critical role in achieving this goal. ${ }^{3}$ 
The unusual structural and chemical properties of zeolite beta make it an important material in diverse applications such as catalytic cracking, isomerization, aromatic alkylation with alkenes, and isobutene alkylation with $n$-butene. ${ }^{4}$ Although one of the first zeolites ever synthesized, its structure has only recently been adequately elucidated because it consists of a disordered intergrowth of different polytypes. ${ }^{5,6}$ Its three distinct polytypes, labelled A, B and C (corresponding to framework types BEA, BEB, and BEC, respectively), have different structures, but these differ only in the stacking sequence of their periodic building units, forming three-dimensional networks of 12-membered ring (12MR) channels connected by smaller 4-, 5and 6-membered rings. Their structures are compared in Fig. 1.

The different polytype structures of zeolite beta can be described in terms of the stacking arrangement of layers of the 12MR channels: ababab in BEA, giving an enantiomorphic tetragonal unit cell; $a b c a b c$ in BEB, with monoclinic symmetry and no chirality; and aaaaaa in $\mathrm{BEC}$, which also has cubic symmetry, but is non-chiral. ${ }^{7} \mathrm{BEA}$ is of particular interest because of its potential for enantioselective catalysis and associated applications, but this is hampered by the difficulty of isolating it from the other polytypes. While the intergrowth of BEA and BEB layers in synthesized crystals does not affect the connectivity of the channels in two crystallographic directions, in the direction of stack faulting, i.e., perpendicular to the multimembered ring layers, the channels become more tortuous than in either of the isolated ideal frameworks, which may affect their permeability to different organic molecules and even their catalytic behavior. An understanding of the formation mechanisms and relative stabilities of the different polytypes may aid in the search for techniques of synthesizing BEA-rich materials with the desired $\mathrm{Si} / \mathrm{Al}$ ratios and counter-cation contents. 
In practice, many zeolites can only be synthesized using organic structure-directing agents (OSDAs) such as quaternary ammonium compounds. In the case of zeolite beta, some OSDAs, if chosen carefully, can increase the proportion of a given polytype,${ }^{8-10}$ but the OSDA must be removed afterwards by calcination before the zeolite can be used. This often leads to microscopic cracks and other defects (such as dealumination, distorted-ring formations, and residual organic impurities) in the zeolite crystals, which also strongly affect the performance of the catalyst or separation membrane in which they are used. ${ }^{11,12}$

Growth of zeolites from seed particles without using an OSDA is an attractive alternative for producing commercially useful zeolites. This method has recently been applied successfully to a number of zeolite systems. ${ }^{11-17}$ Zeolite beta particles synthesized without the use of an OSDA exhibit characteristic crystal habits with a truncated octahedral morphology similar to that of tschernichite, a natural analog of zeolite beta. ${ }^{18}$ Moreover, nuclear magnetic resonance and specific surface area measurements suggest such particles contain far fewer defects than OSDAderived zeolite beta. ${ }^{14,15}$ Consequently, OSDA-free zeolites generally exhibit excellent durability and thermal stability. ${ }^{19}$

In this study, we combine X-ray diffraction (XRD) and electron microscopy analysis of zeolite beta crystals with first-principles calculations of the pure polytype structures in an effort to better understand the complex atomic-scale and microstructural features of this material. OSDA-free zeolite beta crystals were chosen for this because their high crystallinity enables the stacking sequences and hence regions of different polytypes to be identified readily from high-resolution transmission electron microscopy (HRTEM) images. By doing this over a wide region for a large number of crystals, abundance ratios of BEA and BEB polytypes, as well as their distributions, 
could be determined directly. Comparison of these results with macroscale XRD analysis and theoretical calculations suggests that crystals in this system grow by a stochastic mechanism.

\section{Methods}

High-quality zeolite beta crystals were synthesized by adding calcined OSDA-derived beta seeds $(\mathrm{Si} / \mathrm{Al}=12.0 ; \mathrm{Na} / \mathrm{Al}=0.04) 100 \sim 400 \mathrm{~nm}$ in diameter to an OSDA-free $\mathrm{Na}$ aluminosilicate gel with molar composition $13 \mathrm{Na}_{2} \mathrm{O}: \mathrm{Al}_{2} \mathrm{O}_{3}: 40 \mathrm{SiO}_{2}: 1000 \mathrm{H}_{2} \mathrm{O} \cdot{ }^{15,20}$ Hydrothermal treatment was performed at $140{ }^{\circ} \mathrm{C}$ for $40 \mathrm{~h}$ under static conditions and autogeneous pressure. The yield of the fully crystallized sample was ca. $26 \%{ }^{20}$

Powder XRD measurements before and after hydrothermal treatment were performed at room temperature using a D8 Advance Vario1 diffractometer (Bruker AXS) with $\mathrm{Cu}$ K $\alpha$ radiation between 5 and 60 degrees $2 \theta$. The volumetric ratio of BEA to BEB in dehydrated samples was calculated using the DIFFaX + program, ${ }^{21}$ an extension of the original DIFFaX program ${ }^{22}$ that allows a greater number of instrumental, structural, and microstructural parameters to be refined in accordance with the experimental diffraction data.

Crystals were also observed using state-of-the-art high-resolution transmission electron microscopes. HRTEM samples were prepared by dispersing zeolite particles in ethanol, and then depositing them on a $\mathrm{Cu}$ micro-grid mesh without mechanical grinding. As low beam irradiation conditions were necessary to avoid damage to the zeolite structure, observations were performed using a JEOL JEM-3000F microscope at an accelerating voltage of $300 \mathrm{kV}$. Images were processed using the fast Fourier transform method. 
Density functional theory (DFT) calculations of purely siliceous forms of polytypes A, B and C were carried out using the VASP code. ${ }^{23}$ The projector augmented wave method was used with a plane-wave cutoff of $600 \mathrm{eV}$, and electron exchange-correlation effects taken into account using the local density approximation. ${ }^{24}$ For the norm-conserving pseudopotentials, $2 s$ and $2 p$ electrons for $\mathrm{O}$, and $3 s$ and $3 p$ electrons for $\mathrm{Si}$, were explicitly treated as valence electrons. The unit cells of each polytype were used as starting structures, with 192 atoms for polytypes A and B, and 96 atoms in the case of polytype C; 3D periodic boundary conditions were applied in all cases. Atom positions were taken from the Database of Zeolite Structures ${ }^{25}$ for BEA and BEC structures, and Newsam et al. ${ }^{3}$ for the BEB structure. Structural relaxation was terminated when residual forces on all atoms were less than $0.01 \mathrm{eV} / \AA$, and the convergence of the total energies with respect to a cutoff energy up to $800 \mathrm{eV}$ was confirmed to better than $0.015 \mathrm{eV} / \mathrm{f} . \mathrm{u}$. Numerical integration was carried out at the gamma point only owing to the large unit cells involved.

\section{Results and Discussion}

XRD spectra of the powders are shown in Fig. 2. The XRD pattern before dehydration exhibits a remarkably large and broad peak at $2 \theta=7.6^{\circ}$ (Fig. 2a), consistent with the crystals containing roughly equal proportions of BEA and BEB polytypes. ${ }^{19,26}$ After complete dehydration, the intensity of this peak decreases in comparison to the higher angle peaks as the strongly X-ray absorbing adsorbed $\mathrm{H}_{2} \mathrm{O}$ is lost (Fig. 2b). The increased intensity and sharpness of the other peaks also confirm that the OSDA-free beta powder remained crystalline after dehydration. 
Both transmission electron microscopy and scanning electron microscopy confirmed that pyramid-shaped zeolite beta crystals a few hundred nanometers in size formed under the same OSDA-free conditions as reported previously. ${ }^{8}$ These nanocrystals agglomerate to form secondary particles about a micron in size. A typical scanning electron micrograph of the zeolite beta crystals is shown in Fig. 3, and a representative HRTEM micrograph of the apices of the zeolite beta nanocrystals is shown in Fig. 4. Several different regions of the particles were observed, and the excellent resolution of the HRTEM images obtained allowed the positions of framework atoms ( $\mathrm{Si}$ or $\mathrm{Al}$ ) as well as the associated ring and channel structures to be identified individually.

Zeolite beta synthesized using dimethyldibenzylammonium as the OSDA typically contains many structural defects within multimembered ring layers, such as double pores. ${ }^{8}$ In contrast, the zeolite beta crystals synthesized in this study without OSDA were found to exhibit high crystallinity, with no double-pore channels or other intracrystalline defects observed apart from at domain boundaries. A typical boundary between two domains is indicated in Fig. 4; such boundaries appear to be a result of domains with different stacking sequences growing epitaxially from different positions on a $\{001\}$ surface. The formation of different domains produces the pyramid-shaped crystallites that constitute the fractal-like particle morphologies in Fig. 3. The observed epitaxial morphology of the beta crystals is also consistent with the composite building unit mechanism of crystallization from zeolite beta seeds proposed previously. ${ }^{17,20}$

Regions of different layer stackings of various thicknesses can also be readily distinguished within each domain in the HRTEM micrograph of Fig. 4, corresponding to subdomains of BEA and BEB polytypes. The structural units of both polytypes are identical, and layers of these are 
labelled as either oriented to the right (+) or left (-). A +-+ (or equivalently -+-) sequence corresponds to the BEA structure, while +++ (or ---) corresponds to a BEB structure; an example sequence is shown schematically in Fig. 5. The number of contiguous BEB and BEA stacks can thus be counted directly based on the switch in orientation of these multimembered ring layers. Applying this direct method to a number of micrographs (with a total of 482 layers), the ratio of BEA:BEB stacks was calculated to be 45:55. The maximum subdomain thickness observed was five layers for BEA and six layers for BEB. The results of this analysis are summarized in Table 1.

The volume ratio of $\mathrm{BEA}$ to $\mathrm{BEB}$ obtained from $\mathrm{DIFFaX}+$ calculations was found to vary from about 44:56 to 48:52, meaning that the amount of BEB was always slightly greater than that of BEA. This is close to the abundance ratio calculated directly from HRTEM images. Since a similar ratio is found for the crystalline regions in Fig. 4, this relatively small region can be considered representative of the sample as a whole.

The abundance ratio of BEA to BEB will be related to the stacking fault probability because the switch from one polytype to the other depends only on the orientation of a single stacking layer. Assuming that crystal growth in the [001] direction proceeds by sequential precipitation of multimembered chains in a purely stochastic fashion, the probability of a BEA subdomain of thickness $k$ being formed, ${ }_{k} P_{\mathrm{A}}$ (i.e., $k$ sequences of $+/$ - or $-/+$ units), can thus be expressed in terms of the measured abundances as

$$
{ }_{k} P_{\mathrm{A}}=1 / 2 \times\left(P_{\mathrm{A}}\right)^{k-1} \times P_{\mathrm{B}}=1 / 2 \times 0.45^{k-1} \times 0.55
$$

where $P_{\mathrm{A}}$ is the probability of a single BEA stack forming (i.e., the new layer is oriented in the opposite direction to the layer below), and $P_{\mathrm{B}}$ is the probability of a single BEB layer forming 
(i.e., the new layer has the same orientation as the layer below). Similarly ${ }_{k} P_{\mathrm{B}}$, the probability of $k$ BEB stacks being formed (i.e., $k$ sequences of $+/-$ or $-/+$ units), is given by

$$
{ }_{k} P_{\mathrm{B}}=1 / 2 \times\left(P_{\mathrm{B}}\right)^{k} \times P_{\mathrm{A}}=1 / 2 \times 0.55^{k-1} \times 0.45 .
$$

Combining equations (1) and (2) gives the probability ${ }_{k} P_{\mathrm{A}+\mathrm{B}}$ of forming a contiguous sequence of $k$ stacks of either polytype:

$$
{ }_{k} P_{\mathrm{A}+\mathrm{B}}={ }_{k} P_{\mathrm{A}}+{ }_{k} P_{\mathrm{B}}=1 / 2 \times\left(0.45^{k-1} \times 0.55+0.55^{k-1} \times 0.45\right) .
$$

Plots of the probabilities that subdomains will be of a certain thickness, calculated using the results in Table 1, are presented in Fig. 6. Fig. 6a and 6b show the relationships between the number of contiguous stacks and their (normalized) probability of occurrence for BEA and BEB respectively, while Fig. $6 \mathrm{c}$ shows the probability that a subdomain of either polytype will have a certain thickness. For comparison, distributions expected if the stacking sequence varied stochastically based on simple probability theory (eq. 3) are shown as lines in each case. The correlation between the theoretical curves and actual experimental results is excellent.

At the atomic level, whether BEA or BEB regions are formed depends on the change in stacking orientation of multimembered ring chains forming on the (001) crystal plane at the tips of the crystal in the regions indicated by arrows in Fig. 4. Figure 6 shows that the probability that a new layer on the (001) surface will increase the subdomain thickness decreases steadily as the thickness increases, irrespective of the polytype. Conversely, the likelihood that the next layer initiates a new stacking sequence (subdomain) increases with increasing subdomain thickness in accordance with the probabilities $\left(P_{A}\right.$ and $\left.P_{B}\right)$ based on the abundance ratio of the two polytypes. The probability that the subdomain will only be one layer thick is close to $50 \%$ (Fig. 6c). This 
strongly suggests that the multilayer structure is formed by an essentially stochastic process when no OSDA is used.

DFT calculations were performed to obtain some insight into why growth of zeolite beta follows a stochastic process. The calculations reproduced the experimental lattice parameters of each of the three polytypes to within $0.9 \%$. The lattice energies of BEA and BEB were found to differ by less than $0.4 \mathrm{meV}$ per $\mathrm{SiO}_{2}$ formula unit, consistent with earlier forcefield calculations. ${ }^{27} \mathrm{In}$ contrast, BEC, which contains double four ring units with highly strained Si-O-Si bonds, has a lattice energy about $100 \mathrm{meV}$ higher, and thus is significantly less stable. This explains why no BEC regions were observed during HRTEM analysis (e.g., Fig. 4). Within the margin of error of the calculation, therefore, the BEA and BEB structures can be assumed to be equally stable, i.e., neither framework is more thermodynamically favored over the other, so zeolite beta grown under equilibrium or close-to-equilibrium conditions would be expected to consist of $50 \%$ of both polytypes, all else being equal. The computational model, however, assumed a composition of $100 \% \mathrm{SiO}_{2}$, leaving open the possibility that there is a slight energy difference between the BEA and BEB polytypes in the experimentally produced material depending on the distribution and concentration of $\mathrm{Al}$ atoms and the type of compensating cations. ${ }^{28}$ The deviation of the measured abundance ratio from 1:1 (50:50) may also stem from other (kinetic) factors, e.g., chirality effects in solution, or the nature of absorbed species, that affect the orientation of multimembered ring chain layers depositing on crystal surfaces during synthesis. Indeed, it is known that careful control of synthesis conditions and selection of the OSDA can alter the BEA:BEB ratio. ${ }^{6}$ In any case, the DFT results are consistent with the intrinsic disorder in stacking sequences in zeolite beta, and the difficulty of isolating the two crystal phases from each other. Further work is needed to identify the main cause for the different abundances of the two 
polytypes within individual zeolite beta grains, which should provide clues as to how to control more precisely their relative abundances in materials for practical applications.

\section{Conclusions}

We have successfully prepared highly crystalline zeolite beta particles without the use of an OSDA, and quantified the degree of polytype disorder by direct visualization using HRTEM. The results confirm that particles prepared in this way are highly crystalline, with few intracrystalline defects, in contrast to zeolites synthesized using an OSDA. Epitaxial growth appears to occur through the attachment of fresh material to $\{001\}$ surfaces which crystallizes into zeolite beta-type multimembered rings with one of two possible orientations. The stochastic nature of this process produces a disordered intergrowth of BEA and BEB subdomains with an observed maximum thickness of $5 \sim 6$ stacking layers for a single polytype. The ability to characterize and quantify directly zeolitic structures with atomic resolution is expected to become an increasingly important tool in the characterization and design of microporous structures for a wide-range of catalytic and separation membrane-related applications. 


\section{ACKNOWLEDGMENTS}

The authors thank M. Leoni for use of DIFFaX+. This research was supported by the CREST program of the Japan Science and Technology Agency (JST). Part of this work was also supported by a Grant-in-Aid for Scientific Research (24350110) from the Japan Society for the Promotion of Science (JSPS).

\section{REFERENCES}

[1] S. Mintova, J.-P. Gilson, V. Valtchev, Nanoscale 5 (2013) 6693-703.

[2] W. Zhou, J. Mater. Chem. 18 (2008) 5321-5325.

[3] B. Smit and T.M.L. Maesen, Chem. Rev., 108 (2008) 4125-4184.

[4] T. De Baerdemaeker, B. Yilmaz, U. Müller, M. Feyen, F.-S. Xiao, W. Zhang, T. Tatsumi, H. Gies, X. Bao, D. De Vos, J. Catal. 308 (2013) 73-81.

[2] M.M.J. Treacy, J.M. Newsam, Nature 332 (1988) 249-251.

[3] J.M. Newsam, M.M.J. Treacy, W.T. Koetsier, C.B.D. Gruyter, Proc. R. Soc. A Math. Phys. Eng. Sci. 420 (1988) 375-405.

[4] J.B. Higgins, R.B. LaPierre, J.L. Schlenker, A.C. Rohrman, J.D. Wood, G.T. Kerr, W.J. Rohrbaugh, Zeolites 8 (1988) 446-452.

[5] Á. Cantín, A. Corma, M.J. Díaz-Cabañas, J.L. Jordá, M. Moliner, F. Rey, Angew. Chemie Int. Ed. 45 (2006) 8013-8015. 
[6] A. Corma, M. Moliner, Á. Cantín, M.J. Díaz-Cabañas, J.L. Jordá, D. Zhang, J. Sun, K. Jansson, S. Hovmöller, X. Zou, Chem. Mater. 20 (2008) 3218-3223.

[7] J. Zhu, Y. Zhu, L. Zhu, M. Rigutto, A. van der Made, C. Yang, S. Pan, L. Wang, L. Zhu, Y. Jin, Q. Sun, Q. Wu, X. Meng, D. Zhang, Y. Han, J. Li, Y. Chu, A. Zheng, S. Qiu, X. Zheng, F.S. Xiao, J. Am. Chem. Soc. 136 (2014) 2503-2510.

[8] P.A. Wright, W. Zhou, J. Pérez-Pariente, M. Arranz, J. Am. Chem. Soc. 127 (2005) 494495.

[9] B. Mihailova, V. Valtchev, S. Mintova, A.-C. Faust, N. Petkov, T. Bein, Phys. Chem. Chem. Phys. 7 (2005) 2756-2763.

[10] M. Tong, D. Zhang, W. Fan, J. Xu, L. Zhu, W. Guo, W. Yan, J. Yu, S. Qiu, J. Wang, F. Deng, R. Xu, Sci. Rep. 5 (2015) 11521.

[11] J. Sun, G. Zhu, Y. Chen, J. Li, L. Wang, Y. Peng, H. Li, S. Qiu, Micropor. Mesopor. Mater. 102 (2007) 242-248.

[12] B. Xie, J. Song, L. Ren, Y. Ji, J. Li, F.S. Xiao, Chem. Mater. 20 (2008) 4533-4535.

[13] Z. Wu, J. Song, Y. Ji, L. Ren, F.S. Xiao, Chem. Mater. 20 (2008) 357-359.

[14] G. Majano, L. Delmotte, V. Valtchev, S. Mintova, Chem. Mater. 21 (2009) 4184-4191.

[15] Y. Kamimura, W. Chaikittisilp, K. Itabashi, A. Shimojima, T. Okubo, Chem. Asian J. 5 (2010) 2182-2191. 
[16] K. Itabashi, Y. Kamimura, K. Iyoki, A. Shimojima, T. Okubo, J. Am. Chem. Soc. 134 (2012) 11542-11549.

[17] K. Iyoki, K. Itabashi, T. Okubo, Micropor. Mesopor. Mater. 189 (2014) 22-30.

[18] R.C. Boggs, D.G. Howard, J.V. Smith, G.L. Klein, Am. Mineral. 78 (1993) 822-826.

[19] Y. Kubota, K. Itabashi, S. Inagaki, Y. Nishita, R. Komatsu, Y. Tsuboi, S. Shinoda, T. Okubo, Chem. Mater. 26 (2014) 1250-1259.

[20] Y. Kamimura, S. Tanahashi, K. Itabashi, A. Sugawara, T. Wakihara, A. Shimojima, T. Okubo, J. Phys. Chem. C 115 (2011) 744-750.

[21] M. Leoni, A. F. Gualtieri, N. Roveri, J. Appl. Crystallogr. 37 (2004) 166-173.

[22] M.M.J. Treacy, J.M. Newsam, M.W. Deem, Proc. R. Soc. London. Ser. A 433 (1991) 499-520.

[23] G. Kresse, Phys. Rev. B 54 (1996) 11169-11186.

[24] P.E. Blöchl, Phys. Rev. B 50 (1994) 17953-17978.

[25] http://www.iza-structure.org/databases/

[26] T. Willhammar, X. Zou, Z. Kristallogr. 228 (2013) 11-27

[27] S.M. Tomlinson, R.A. Jackson, C.R.A. Catlow, J. Chem. Soc. Chem. Commun. (1990) 813-816. 
[28] R. Hajjar, Y. Millot, P.P. Man, M. Che, S. Dzwigaj, J. Phys. Chem. C 112 (2008) 2016720175. 


\section{Figure Captions}

Fig. 1. Crystal structures of zeolite beta polytypes comparing stacking order of the periodic building units (12-ring pore channels): (a) BEA ( $\left.4_{1} 22\right)$, (b) BEB (C12/cl), and (c) BEC $\left(P 4_{2} / m m c\right)$. Dotted lines demarcate a single unit cell in each case.

Fig. 2. Powder X-ray diffraction patterns of OSDA-free zeolite beta (a) before and (b) after dehydration.

Fig. 3. A scanning electron microscope image showing the characteristic size and morphology of zeolite beta crystals synthesized without an organic structure directing agent.

Fig. 4. An HRTEM micrograph of the apices of a crystal viewed down the BEA [010] axis showing subdomains of polytypes A (BEA) and B (BEB) based on sequences of + or - oriented multimembered ring layers. Arrows indicate amorphous regions at two of the larger apices, as well as the boundary between two domains.

Fig. 5. Schematic diagram of different stacking sequences of multimembered ring chains resulting in BEA- or BEB-type subdomains within a zeolite beta crystal.

Fig. 6. (a) Probability of a BEA subdomain of a given thickness forming within a zeolite beta crystal; (b) Probability of a BEB subdomain of a given thickness forming within a zeolite beta crystal; (c) Probability of forming contiguous stacks of either polytype for a given subdomain thickness. In each case dots show the experimental data and the continuous line the values corresponding to a stochastic probability model.

\section{Table caption}

Table 1. Numbers of contiguous stacks of polytypes BEA and BEB measured directly from several HRTEM micrographs 
Figure 1

(a)

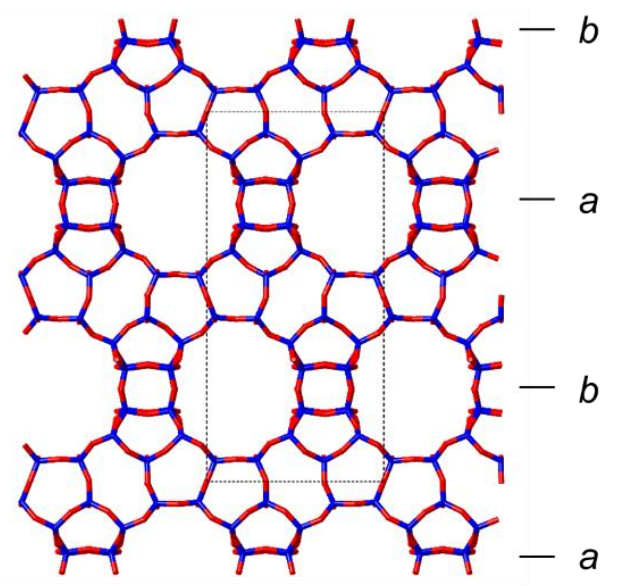

(b)

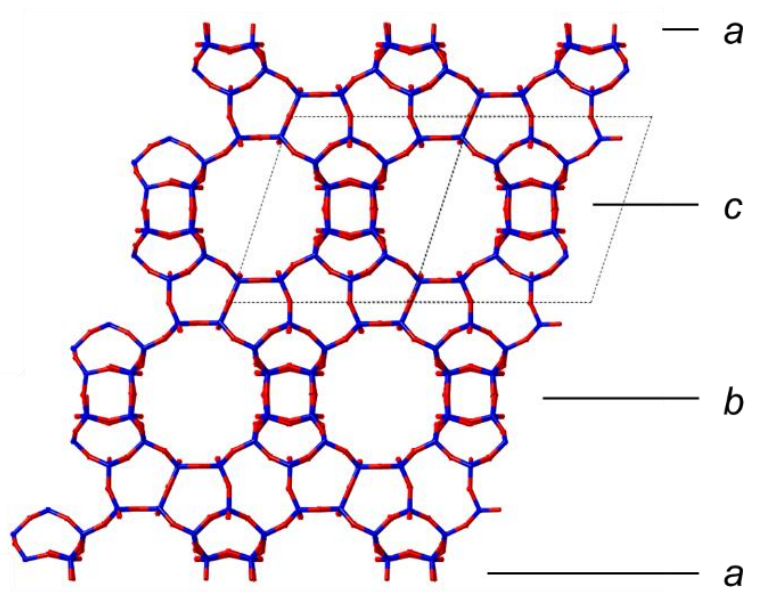

(c)

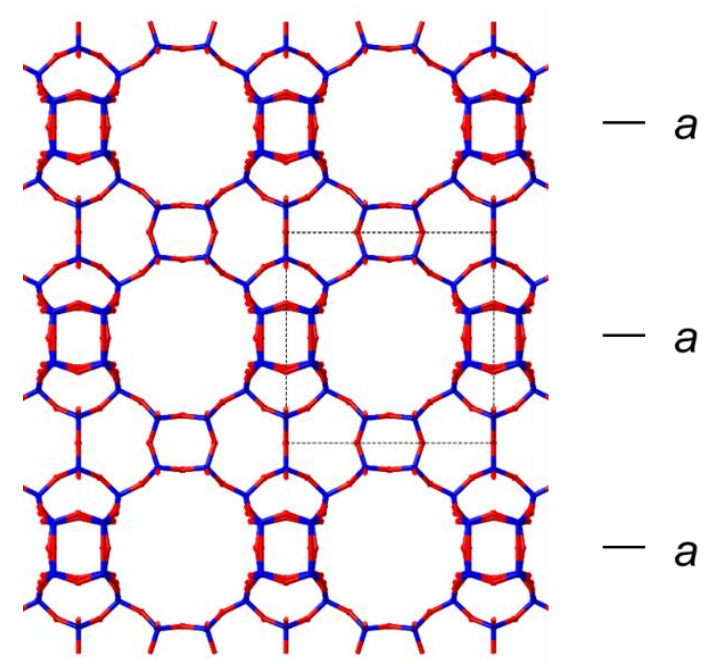


Figure 2

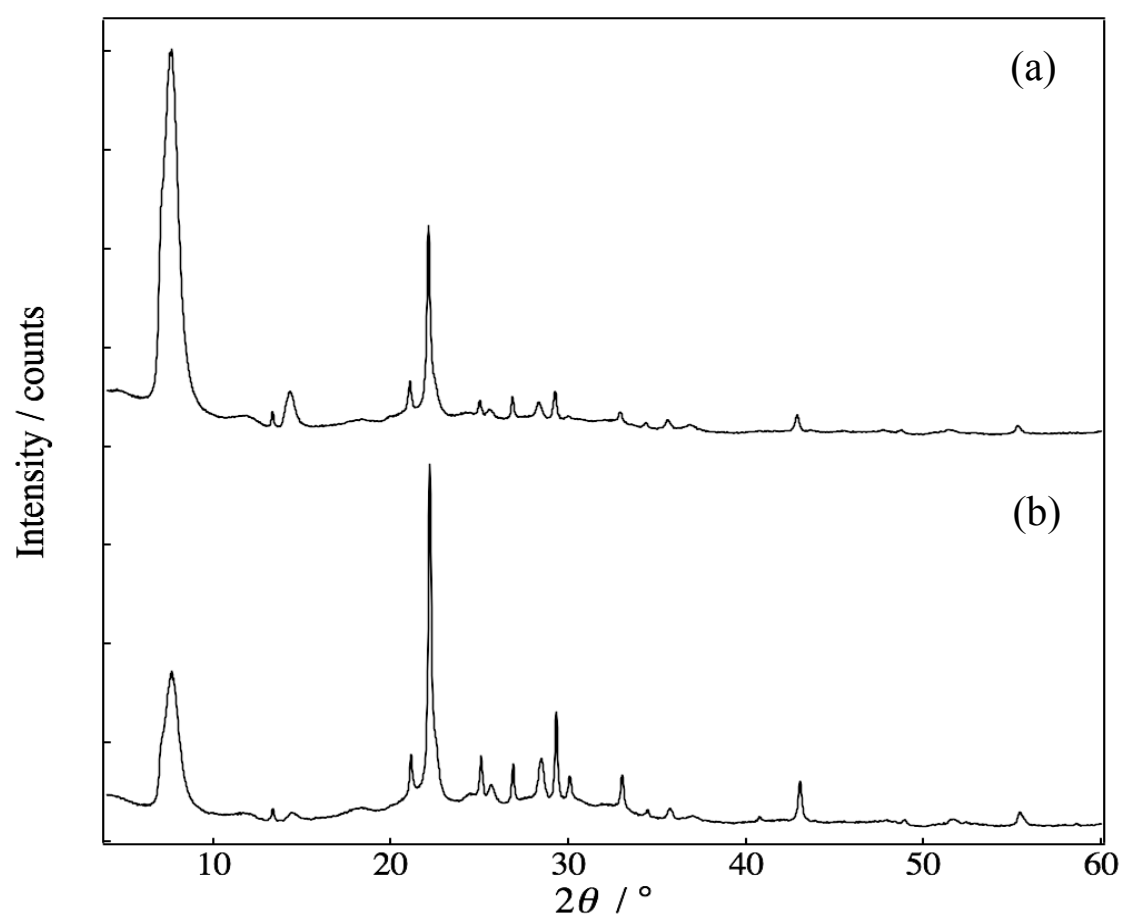


Figure 3

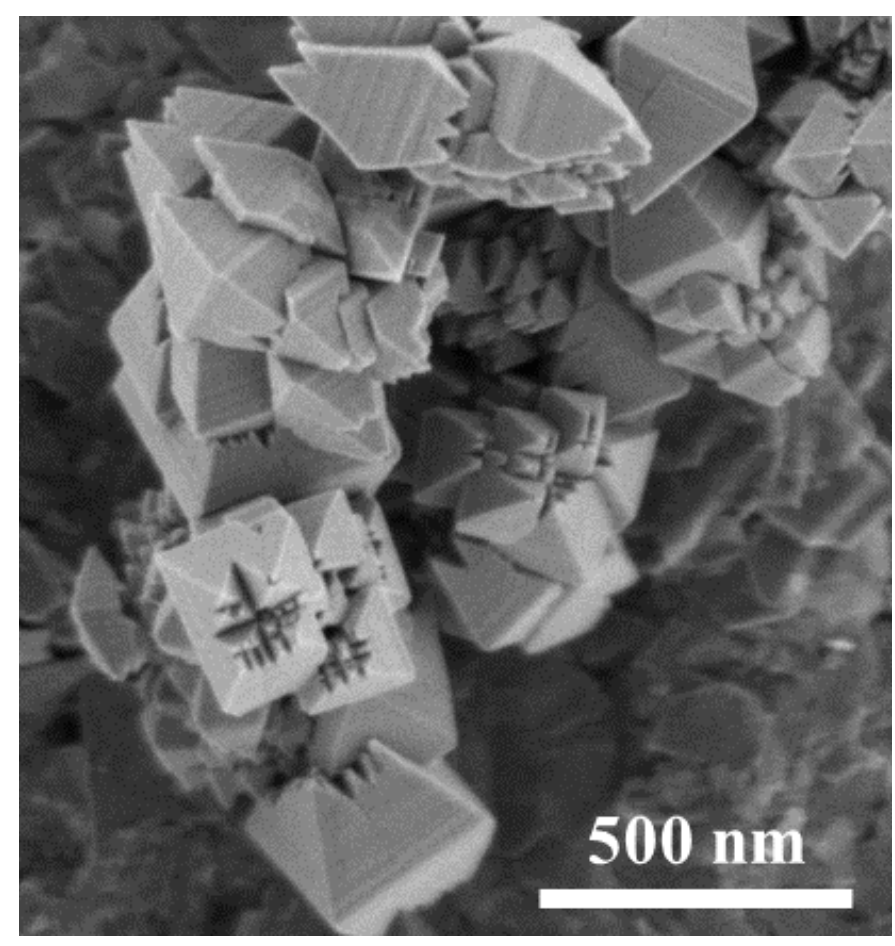


Figure 4

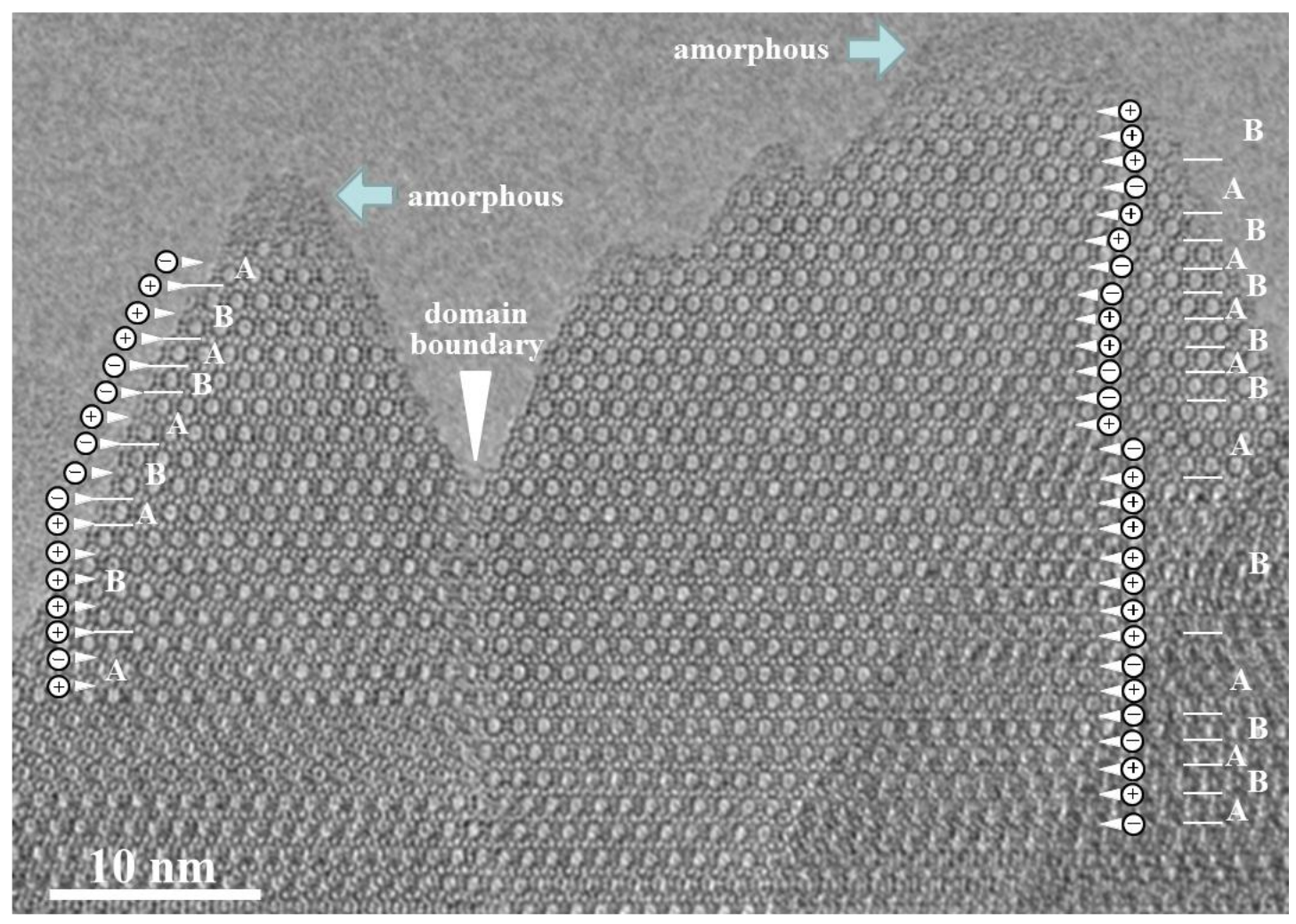


Figure 5

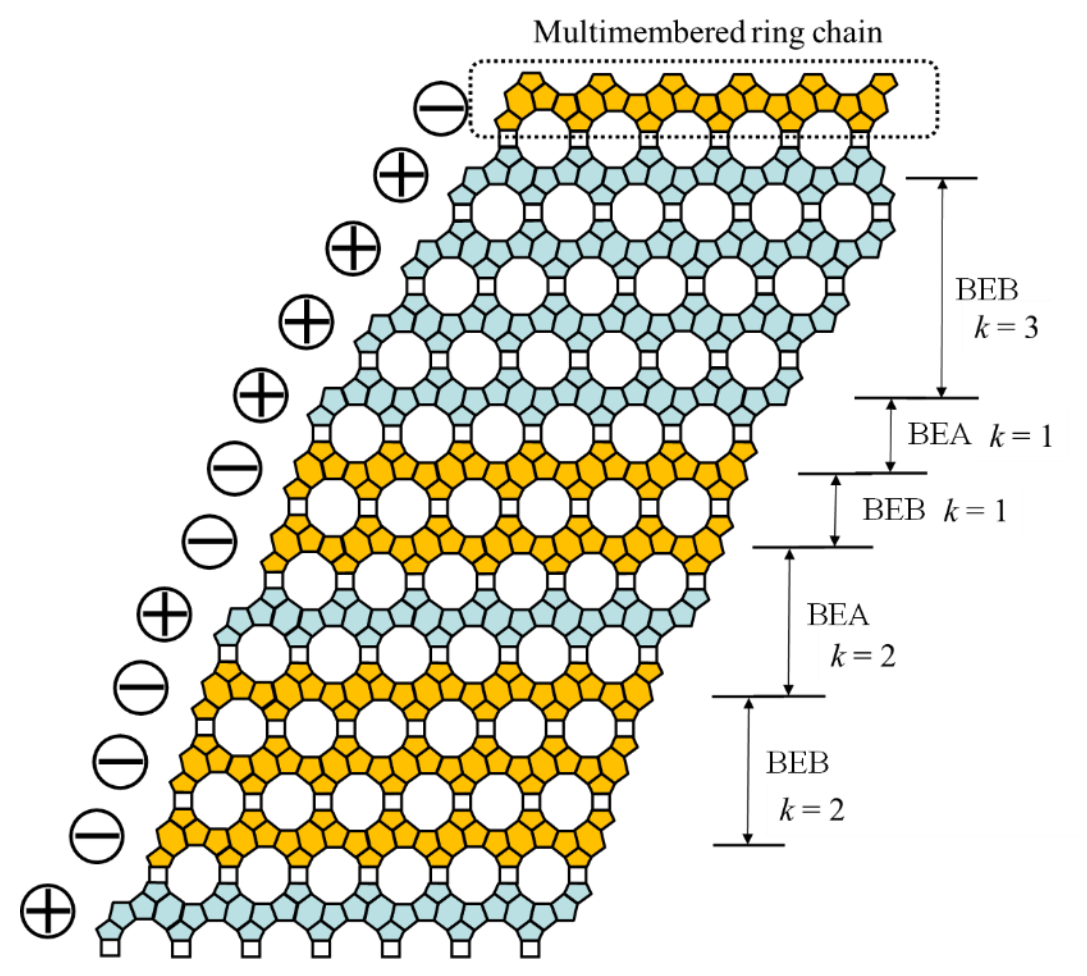


Figure 6

(a)

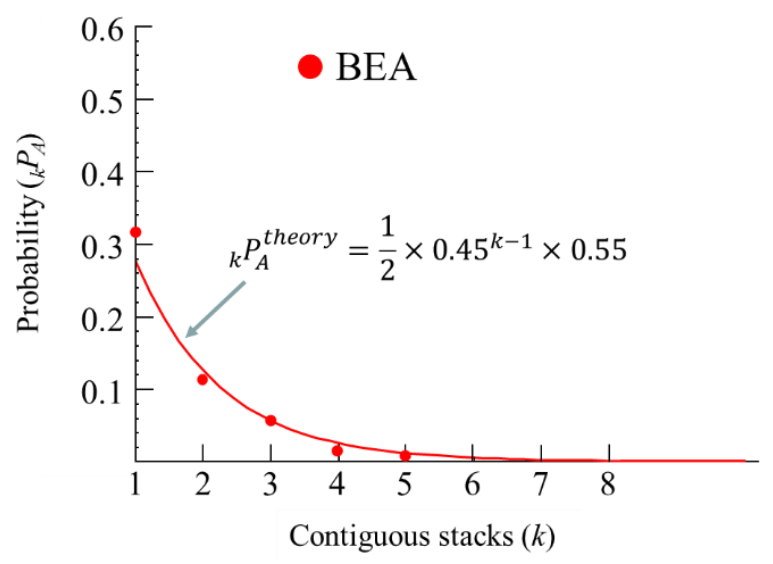

(b)

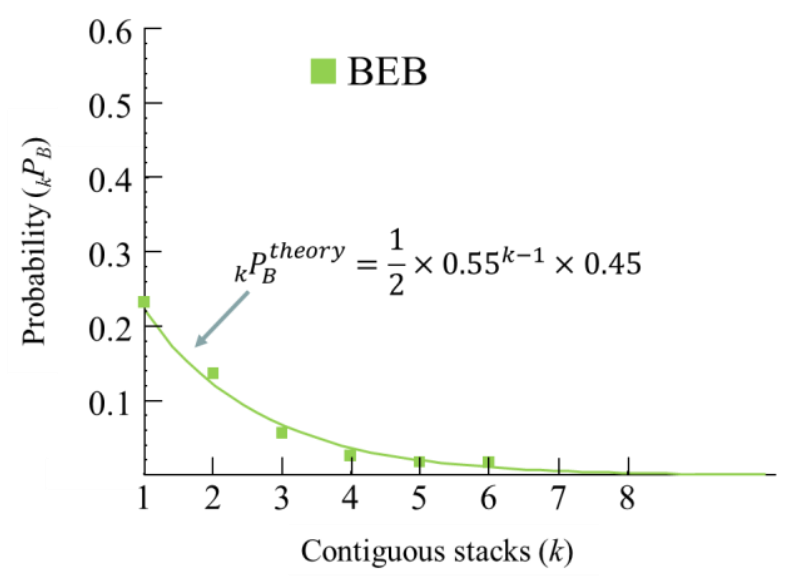

(c)

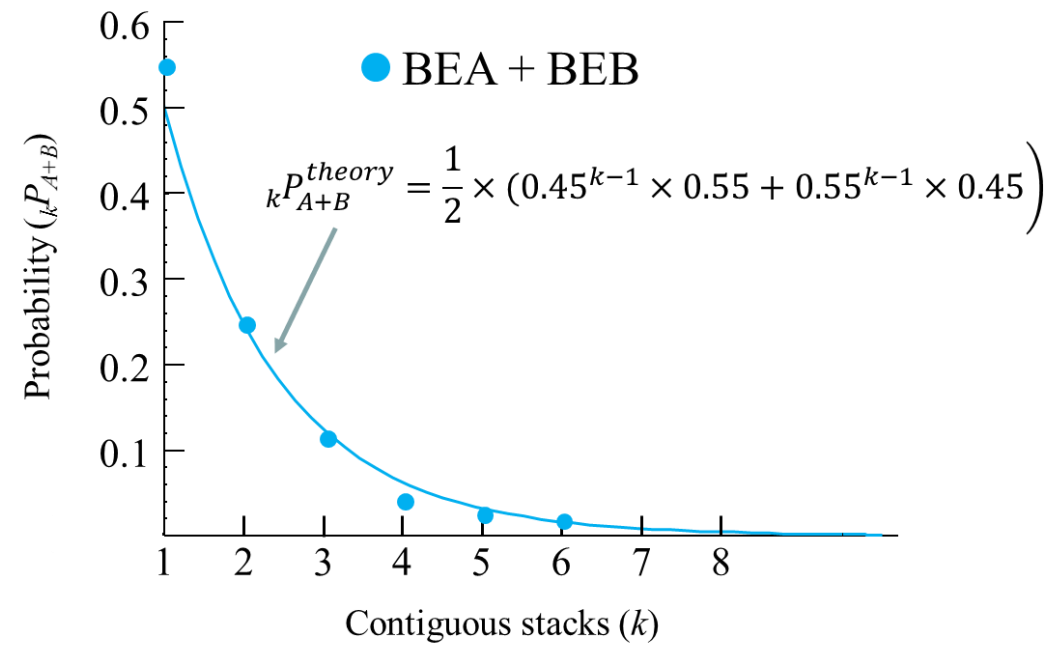


Table 1. Numbers of contiguous stacks of polytypes BEA and BEB measured directly from several HRTEM micrographs.

\begin{tabular}{|c|c|c|c|}
\hline $\begin{array}{c}\text { Number of } \\
\text { contiguous stacks }\end{array}$ & BEA & BEB & BEA+BEB \\
\hline 1 & 85 & 63 & 148 \\
\hline 2 & 30 & 37 & 67 \\
\hline 3 & 15 & 16 & 31 \\
\hline 4 & 4 & 7 & 11 \\
\hline 5 & 2 & 5 & 7 \\
\hline 6 & 0 & 5 & 5 \\
\hline Total number of \\
layers
\end{tabular}




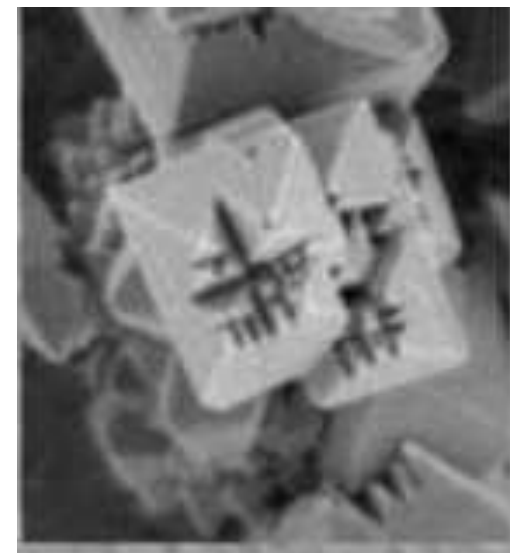

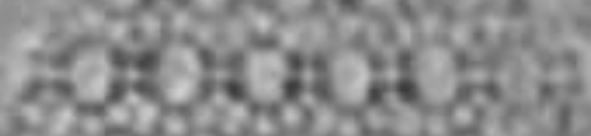

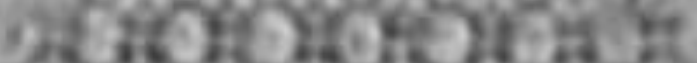

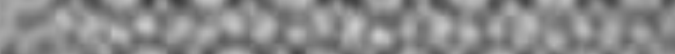

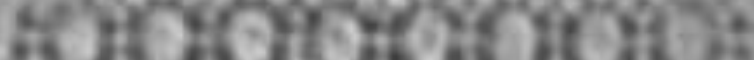

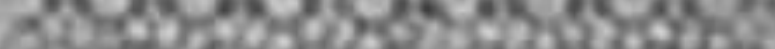

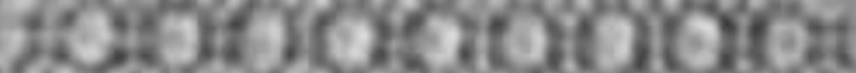

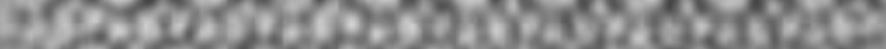

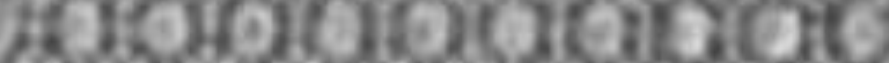

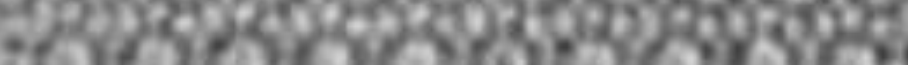

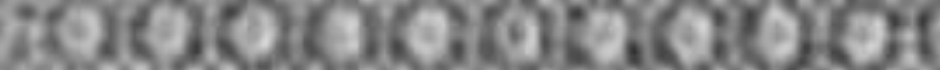

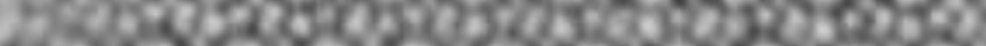

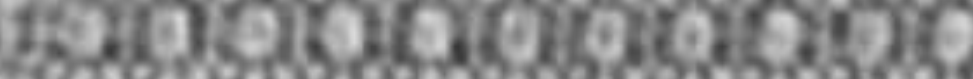

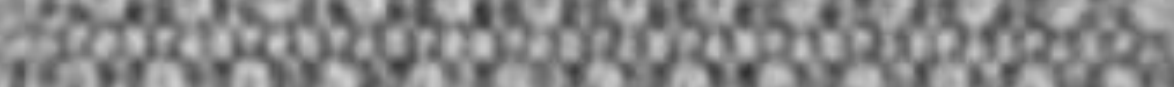

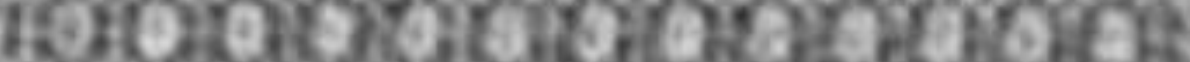

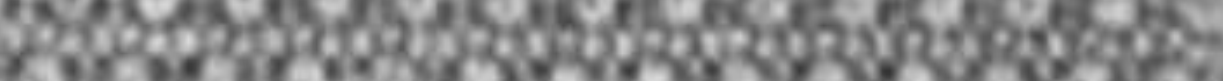

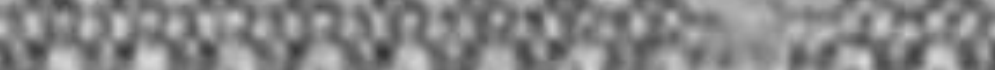

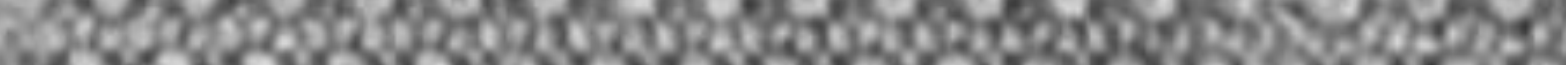

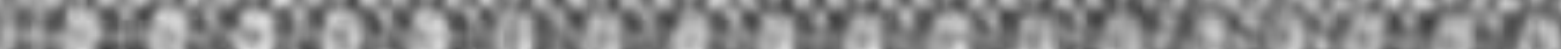
H.

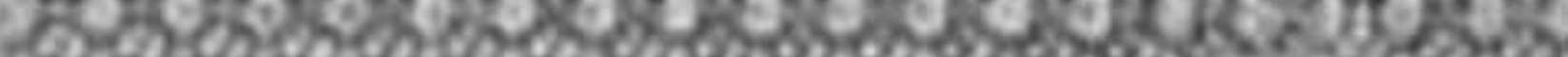

I

if

a

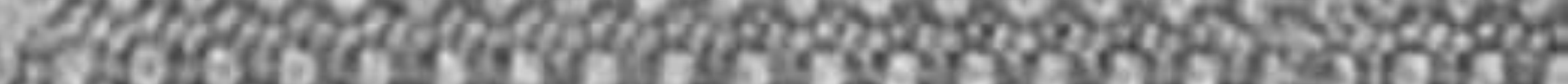

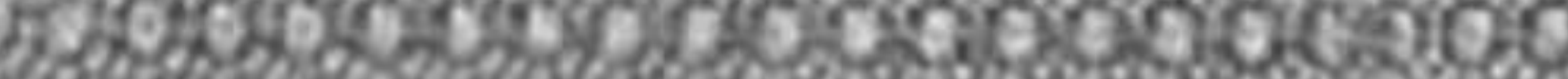

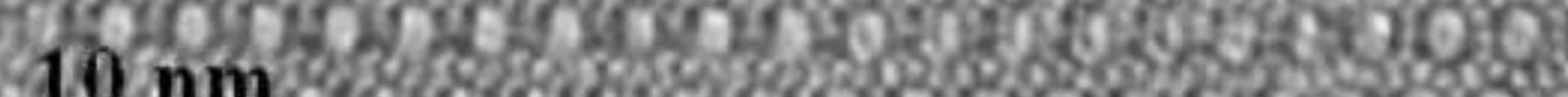

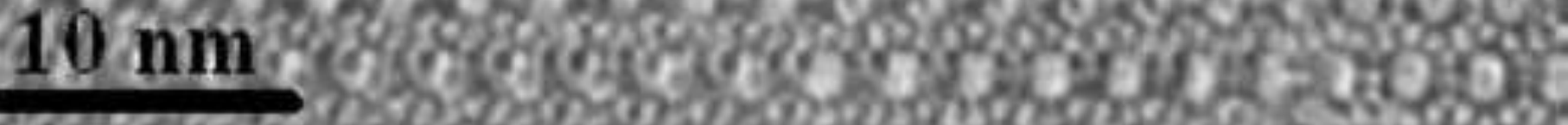

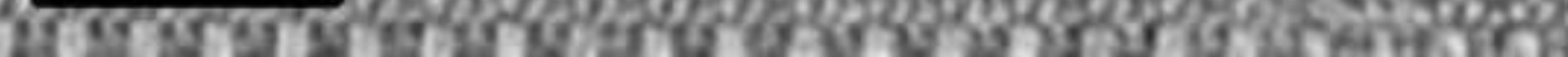

